\title{
STRESS-DILATANCY FOR SOILS. PART IV: EXPERIMENTAL VALIDATION FOR SIMPLE SHEAR CONDITIONS
}

\author{
ZENON SZYPCIO \\ Department of Civil and Environmental Engineering, \\ Białystok University of Technology, Białystok, Poland, e-mail: z.szypcio@pb.edu.pl
}

\begin{abstract}
This paper validates the frictional state theory using published experimental data from simple, direct and ring shear tests. Simple shear is treated as a special case of plane strain conditions. In order to define complete stress and strain, additional assumptions are made: in the direct shear and ring shear tests, simple shear is assumed to occur in the shear band. For $\Phi^{o}=\Phi_{c v}^{\prime}=\Phi_{r}^{\prime}$, the stress-dilatancy relationship obtained from the frictional state theory is similar to the relationships proposed by Taylor and Bolton. Further experiments, especially those that use a hollow cylindrical shear apparatus, are necessary to fully validate the frictional state theory in simple shear conditions.
\end{abstract}

Key words: soils, dilatancy, critical state, frictional state, simple shear

\section{INTRODUCTION}

Simple shear is directly achieved in the two basic designs for a simple shear apparatus, which are commonly known as the Cambridge and Norwegian Geotechnical Institute (NGI) types. In the Cambridge type, a rectangular sample is completely surrounded by rigid platens. In the Norwegian apparatus, a cylindrical specimen is sealed in a rubber sheath reinforced by spiral wire to allow pore pressure measurement; the test can be either drained or undrained [30]. During a conventional simple shear test, the deformation of the whole sample is reasonably uniform, the cross section changes from a rectangle to a parallelogram, and only normal and shear stresses are measured on the horizontal plane. The stress state is not possible to completely define in a soil sample ([1], [38]).

The direct shear test is conventionally used for measuring the strength parameters of soils. The nonuniformity of the soil sample deformation is much greater in the direct shear test than in the simple shear test. The shear stress and normal stress on the shear plane (central horizontal plane) are determined from the vertical load acting on the top platen and the horizontal reaction force measured outside together with the changing cross-sectional area of the shear plane. Conventionally, the change in the total sample height is also measured during the test.
The stress and strain non-uniformities within direct shear specimens can be recognized from the experimental ([26], [28]) continuum approach using finite element analysis ([2], [23]) and the microscale approach using discrete element modelling (DEM) analysis ([8], [37], [40]). Strong non-uniformity of the stresses and strains is shown before the peak; however, at failure, the deformation and stress in the failure zone are surprisingly uniform [13]. The strength parameters of the soil obtained in simple and direct shear tests are similar [34]. The specimen preparation technique and specimen size can influence the strength parameters obtained from direct shear tests ([3], [6], [22], [39]).

Conventionally, in the ring shear test, an annular specimen is placed in a split box, and a shear force is produced by torsion. The main advantage of this test compared to the direct shear test is the constant size of the shear surface at any value of shear displacement, which allows the residual strength to be determined. In the conventional direct and ring shear tests, the unknown effects of the opening between the top and bottom halves of the shear box and the side-wall friction may seriously affect the test results [24].

Some modifications of the direct and simple shear box ([5], [14], [15], [18], [36]) and ring shear apparatus ([19], [25]) were proposed to eliminate some faults of this device, especially for tests of saturated and unsaturated soils. 
A simple shear test with known stresses and strains can be achieved using a hollow cylindrical torsional shear apparatus ([10]-[12], [27]).

Based on experimental data presented in the literature, this paper presents the validation of the frictional state theory [31]. The simple shear condition is treated as a special case of the plane strain condition [33]. Because the stress and strain during shear are unknown in the conventional simple shear test, additional assumptions are introduced. The angle between the minimum major normal stress direction and the horizontal plane is assumed to be constant and equal to $\pi / 4+\Phi^{o} / 2$; in addition, the horizontal plane is assumed to neither extend nor contract during shearing. The walls of the apparatus are assumed to be perfectly smooth, and the deformation of the sample is assumed to be homogeneous, so that the stress and strain can be defined by the external conditions. The direct and ring shear tests are treated as special cases of the simple shear test. The condition of simple shear occurs in a shear band of unknown thickness. The stress-dilatancy relationship obtained from the frictional state theory is compared with the relationships proposed by Taylor [35] and Bolton [4]. Experimental data on granular soils are analysed.

\section{STRESS-DILATANCY RELATIONSHIP}

The geometry, stress and strain in simple shear conditions are shown in Fig. 1.

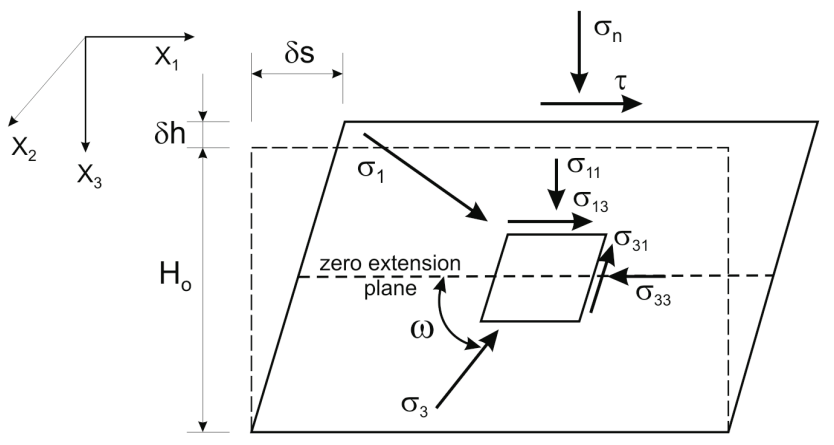

Fig. 1. Geometry, stress and strain in simple shear conditions

An increase in the sample height $(\delta h)$ is assumed to be positive. The horizontal plane is taken to be a zero extension plane, and the angle between the minor principal stress $\left(\sigma_{3}\right)$ direction and the horizontal plane is

$$
\omega=\frac{\pi}{4}+\frac{\Phi^{o}}{2} .
$$

The principal stresses [31] are functions of $p^{\prime}, q$, and $\theta$

$$
\begin{gathered}
\sigma_{1}^{\prime}=p^{\prime}=\frac{2}{3} q \sin \left(\theta-\frac{2}{3} \pi\right), \\
\sigma_{2}^{\prime}=p^{\prime}-\frac{2}{3} q \sin \theta, \\
\sigma_{3}^{\prime}=p^{\prime}-\frac{2}{3} q \sin \left(\theta+\frac{2}{3} \pi\right) .
\end{gathered}
$$

The stress and strain components in this paper are

$$
\begin{gathered}
\sigma_{11}^{\prime}=\frac{1}{2}\left(\sigma_{1}^{\prime}+\sigma_{3}^{\prime}\right)+\frac{1}{2}\left(\sigma_{1}^{\prime}-\sigma_{3}^{\prime}\right) \sin \Phi^{o}, \\
\sigma_{22}^{\prime}=\sigma_{2}^{\prime}, \\
\sigma_{33}^{\prime}=\sigma_{n}^{\prime}=\frac{1}{2}\left(\sigma_{1}^{\prime}+\sigma_{3}^{\prime}\right)-\frac{1}{2}\left(\sigma_{1}^{\prime}-\sigma_{3}^{\prime}\right) \sin \Phi^{o}, \\
\sigma_{13}^{\prime}=\tau=\frac{1}{2}\left(\sigma_{1}^{\prime}-\sigma_{3}^{\prime}\right) \cos \Phi^{o}, \\
\sigma_{12}^{\prime}=\sigma_{23}^{\prime}=0 .
\end{gathered}
$$

Neglecting the elastic increment, we have

$$
\begin{gathered}
\delta \varepsilon_{11}=0, \\
\delta \varepsilon_{22}=\delta \varepsilon_{2}=0, \\
\delta \varepsilon_{33}=\frac{\delta h}{H_{o}}, \\
\delta \varepsilon_{13}=\frac{1}{2} \delta \gamma=\frac{\delta s}{2 H_{o}}, \\
\delta \varepsilon_{12}=\delta \varepsilon_{23}=0 .
\end{gathered}
$$

The volume strain increment is

$$
\delta \varepsilon_{v}=-\frac{\delta h}{H_{o}}
$$

and the shear strain increment is

$$
\delta \varepsilon_{q}=\frac{1}{\sqrt{3}} \frac{\delta s}{H_{o}} \sqrt{1+\frac{4}{3}\left(\frac{\delta h}{\delta s}\right)^{2}} .
$$

Therefore,

$$
D^{p}=D=\frac{\delta \varepsilon_{v}}{\delta \varepsilon_{q}}=-\sqrt{3} \frac{\delta h / \delta s}{\sqrt{1+\frac{4}{3}\left(\frac{\delta h}{\delta s}\right)^{2}}}
$$


or

$$
D=-\frac{3 \sin \psi}{\sqrt{3+\sin ^{2} \psi}}
$$

where the dilatancy angle $(\psi)$ is calculated using the equation

$$
\tan \psi=\frac{\delta h}{\delta s}
$$

Combining equations (2) and (3), after some algebra, we can write

$$
\begin{gathered}
\tau=\frac{\sqrt{3}}{3} q \cos \Phi^{o} \cos \theta, \\
\sigma_{n}^{\prime}=p^{\prime}-\frac{1}{3} q\left\{\sin \theta-\sqrt{3} \sin \Phi^{o} \cos \theta\right\},
\end{gathered}
$$

and the mobilized value of the friction angle $\left(\Phi_{s s}^{\prime}\right)$ can be calculated from the equation

$$
\tan \Phi_{s s}^{\prime}=\frac{\tau}{\sigma_{n}^{\prime}}=\frac{\sqrt{3} \eta \cos \Phi^{o} \cos \theta}{3+\eta\left\{\sin \theta-\sqrt{3} \sin \Phi^{o} \cos \theta\right\}} .
$$

From the frictional state theory [31]

$$
\eta=M_{b}^{o}-A_{b}^{o}\left(\alpha+\beta D^{p}\right)
$$

where

$$
\begin{gathered}
M_{b}^{o}=\frac{3 \sin \Phi^{o}}{\sqrt{3} \cos \theta-\sin \Phi^{o} \sin \theta} \\
A_{b}^{o}=\frac{1}{\cos \left(\theta-\theta_{\varepsilon}\right)}\left\{1-\frac{2}{3} M_{b}^{o} \sin \left(\theta+\frac{2}{3} \pi\right)\right\}
\end{gathered}
$$

for the drained condition,

$$
A_{b}^{o}=\frac{1}{\cos \left(\theta-\theta_{\varepsilon}\right)}\left\{1-\frac{2}{3} M_{b}^{o} \sin \left(\theta-\frac{2}{3} \pi\right)\right\}
$$

for the undrained condition,

$\alpha$ and $\beta$ are frictional state theory parameters. For simple shear conditions, the angle $\left(\theta_{\varepsilon}\right)$ for the assumptions adopted in this paper can be calculated from the equation

$$
\tan \theta_{\varepsilon}=\frac{1}{\sqrt{3}} \frac{\delta h / \delta s}{\sqrt{1+(\delta h / \delta s)^{2}}} .
$$

For convenience, subscript b is omitted on $\theta$ or $\theta_{\varepsilon}$.

The complex equation (12) is the stress-dilatancy equation for the simple shear condition obtained from the frictional state theory [31]. Angle $\Phi^{o}$ for many granular soils can be assumed to be equal to the critical state friction angle $\Phi_{c v}^{\prime}[32]$.
The extended Taylor [35] equation is

$$
\tan \Phi^{\prime}=\tan \Phi_{r}^{\prime}+\frac{\delta h}{\delta s}
$$

and the Bolton (1986) equation is

$$
\Phi^{\prime}=\Phi_{c v}^{\prime}+0.8 \psi
$$

The stress-dilatancy equation for the simple shear condition, as shown in equation (12), is not a function of the sample height $\left(H_{o}\right)$; therefore, this equation can be used for direct and ring shear tests, where simple shear occurs in only a shear band of unknown thickness.

(a)
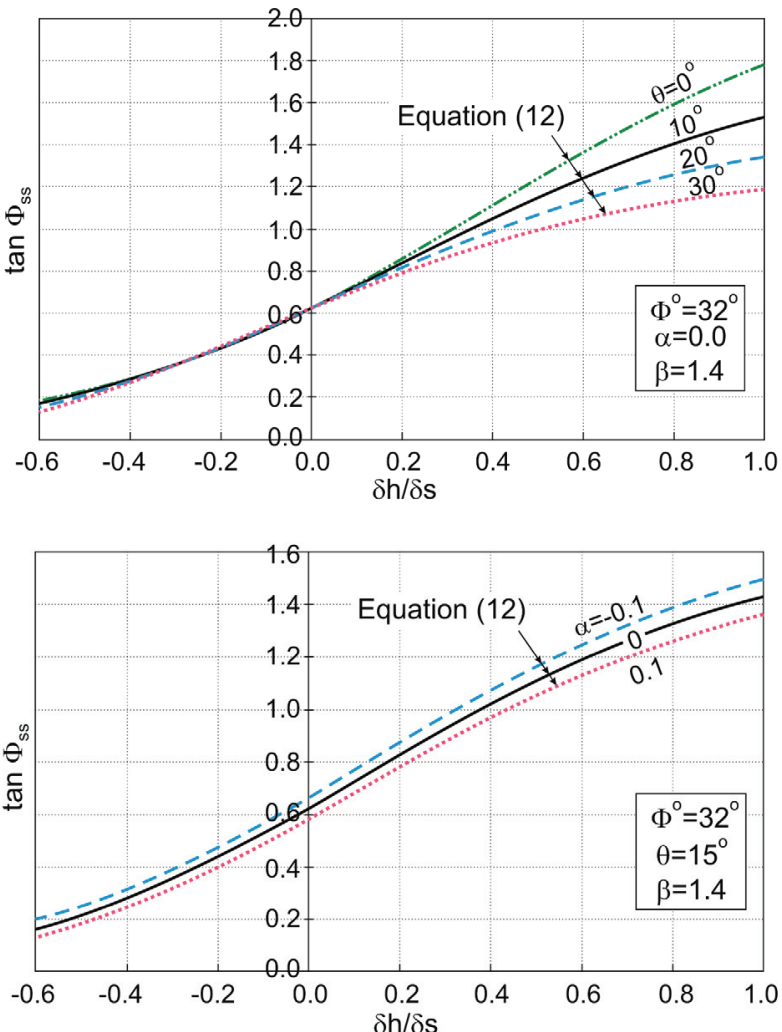

(b)

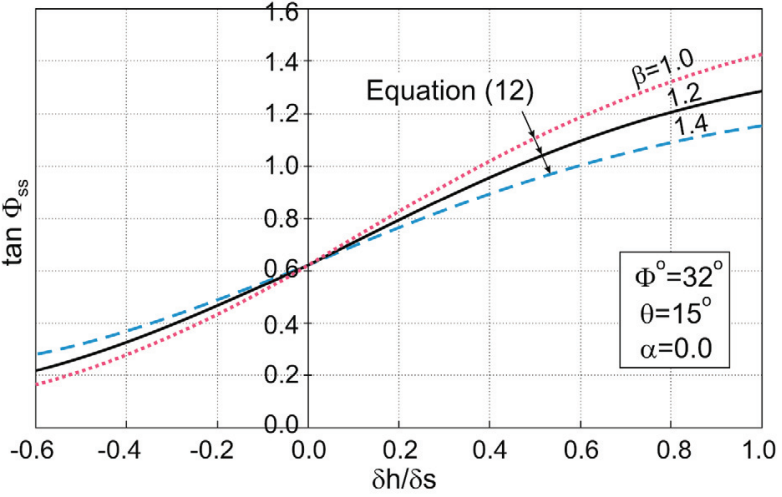

Fig. 2. Influence of $\theta, \alpha$ and $\beta$ on the stress ratio-dilatancy relationship: (a) $\theta=0,10,20$, and 30 degrees; (b) $\alpha=-0.1,0$, and 0.1 ; (c) $\beta=1.0,1.2$, and 1.4 
The influence of the parameters $\theta, \alpha$ and $\beta$ on the stress ratio $\left(\tau / \sigma_{n}^{\prime}=\tan \Phi_{s s}^{\prime}\right)$ are shown in Fig. 2, where $\Phi_{s s}^{\prime}$ is the angle of friction under simple shear.

Angle $\theta$ strongly influences the stress ratiodilatancy relationship for $\delta h / \delta s>0.3$, but the influence is small for $\delta h / \delta s<0.3$, especially for contraction during shear $(\delta h / \delta s<0)$. Parameter $\alpha$ translates the reference curve obtained for $\alpha=0$ upward for $\alpha<0$ and downward for $\alpha>0$. Parameter $\beta$ significantly influences the stress ratio-dilatancy relationship for dilation and contraction during shear.

Figure 3 compares the values of $\Phi_{s s}^{\prime}$ obtained from the frictional state theory [31] and from the theories of Taylor [35] and Bolton [4].

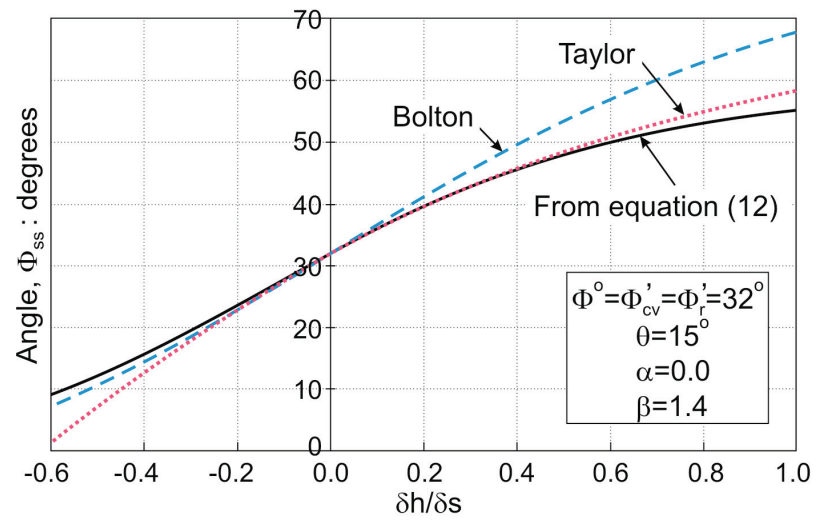

Fig. 3. Comparison of different relationships

Calculations were performed for $\theta=15^{\circ}, \alpha=0$, and $\beta=1.4$, which are typical of granular soils in plane strain conditions [33]. For granular soils, the values of $\Phi_{s s}^{\prime}$ calculated from the frictional state theory are close or very close to the values calculated from the Taylor [35] relationship for $-0.3<\delta h / \delta s$ $<0.8$ and to the Bolton [4] relationship for $-0.6<$ $\delta h / \delta s<0.4$. The Taylor [35] and Bolton [4] relationships can be treated as special cases of the stressdilatancy relationship obtained from the frictional state theory.

The validation of the frictional state theory for simple shear is based on the extensive investigation of Leighton Buzzard sand in a Cambridge type simple shear apparatus performed by Cole [7]. The relationships between $\tan \Phi_{s s}^{\prime}$ and $\delta h / \delta s$ for three selected tests [7] with an initial porosity of $e_{o}=0.755$ and $\sigma_{n}^{\prime}=4.80,21.4$ and $38.65 \mathrm{kPa}$ obtained from the test data analysis quoted by Oda [21] are shown in Fig. 4.

The analysis of the values of the stress ratio $\left(\tau / \sigma_{n}^{\prime}\right.$ $\left.=\tan \Phi_{s s}^{\prime}\right)$ and the changes in the sample height $(\delta h)$ were estimated by high degree polynomials, and the relationships between $\tan \Phi_{s s}^{\prime}$ and $\delta h / \delta s$ were calculated.

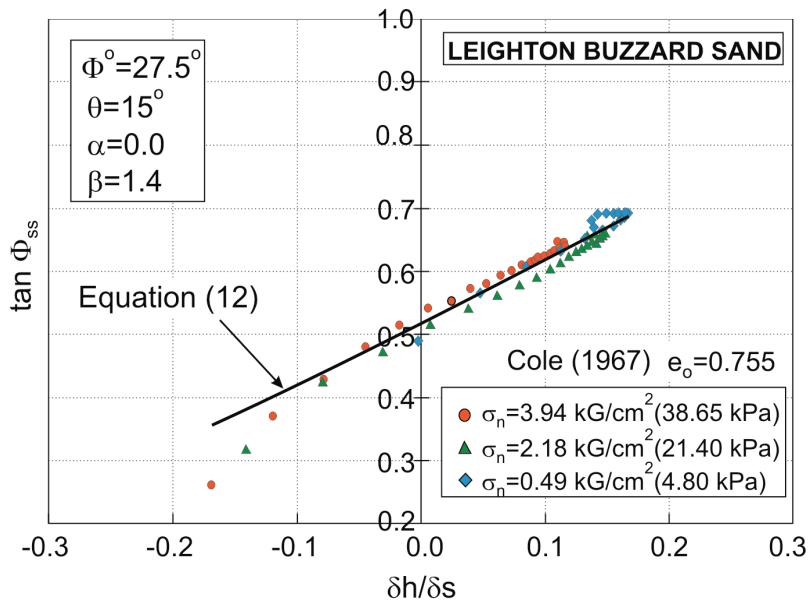

Fig. 4. Influence of the stress level on the stress ratio-dilatancy relationship in simple shear conditions

A good approximation of the experimental data was obtained from equation (12) with $\Phi^{o}=27.5^{\circ}$, $\theta=15^{\circ}, \alpha=0.0$ and $\beta=1.4$. The same calculation procedure was used for three different tests with $\sigma_{n}^{\prime}=21.6 \mathrm{kPa}$ and initial porosities of $e_{o}=0.549$, 0.645 and 0.755 . The best fit of the experimental data [7] was obtained for $\Phi^{\circ}=27.5^{\circ}$ and $\theta=15^{\circ}$ but for different values of $\alpha$ and $\beta$ (Fig. 5).

The theoretically estimated value of the stress ratio $\left(\tau / \sigma_{n}^{\prime}\right)_{o}$ at $\delta \varepsilon_{v} / \delta \gamma=0(\delta h / \delta s=0)$ obtained by Oda [21] is 0.51 . Therefore, the critical frictional state angle value estimated by Oda [21] is $27.0^{\circ}$. The critical state angle of Leighton Buzzard sand is $\Phi_{c v}^{\prime}=35^{\circ}$ ([7], [20]), so $\Phi^{o} \ll \Phi_{c v}^{\prime}$ for Leighton Buzzard sand.

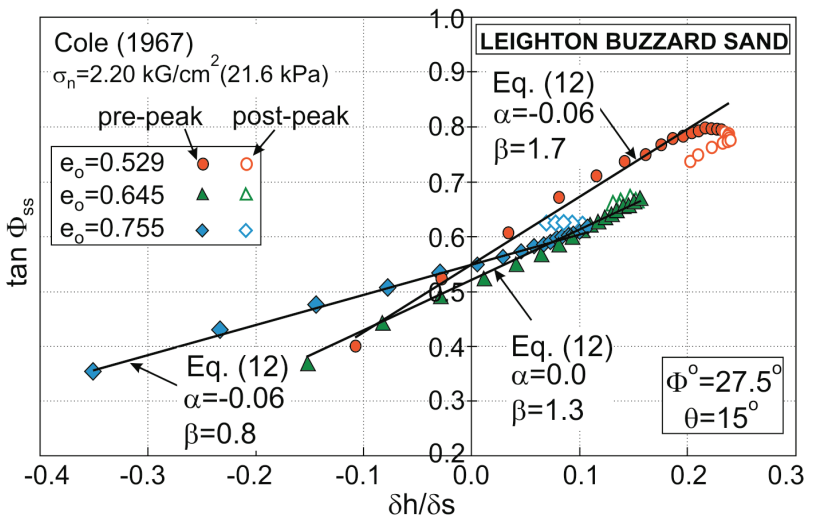

Fig. 5. Influence of the initial porosity on the stress ratio-dilatancy relationship in simple shear conditions

The different values of $\alpha$ and $\beta$ obtained from the approximation may be caused by varying test condi- 
tions or different initial structures of the soil samples as a result of the sample preparation method.

Gutierrez and Wang [9], analysing the simple shear experimental data of Leighton Buzzard sand, showed that using the non-coaxial version of Rowe's stress-dilatancy relation provided a good approximation of the experimental relation $t / s-\delta \mathrm{v} / \delta \gamma$ (Fig. 6), where

$$
\begin{gathered}
t=\left(\sigma_{1}^{\prime}-\sigma_{3}^{\prime}\right) / 2, \\
s=\left(\sigma_{1}^{\prime}+\sigma_{3}^{\prime}\right) / 2, \\
\frac{t}{s}=\frac{\sigma_{1}^{\prime} / \sigma_{3}^{\prime}-1}{\sigma_{1}^{\prime} / \sigma_{3}^{\prime}+1}, \\
\frac{\sigma_{1}^{\prime}}{\sigma_{3}^{\prime}}=\frac{3-2 \eta \sin \left(\theta-\frac{2}{3} \pi\right)}{3-2 \eta \sin \left(\theta+\frac{2}{3} \pi\right)}, \\
\delta \gamma=\left\{\left(\delta \varepsilon_{11}-\delta \varepsilon_{33}\right)^{2}+4 \delta \varepsilon_{13}^{2}\right\}^{1 / 2}, \\
\delta \varepsilon_{v} / \delta \gamma=\delta h / \delta s .
\end{gathered}
$$

Using equation (23) with $\Phi^{o}=27.5^{\circ}, \theta=15^{\circ}, \alpha=$ 0.10 , and $\beta=1.4$, a very good approximation of the experimental data was obtained (Fig. 6).

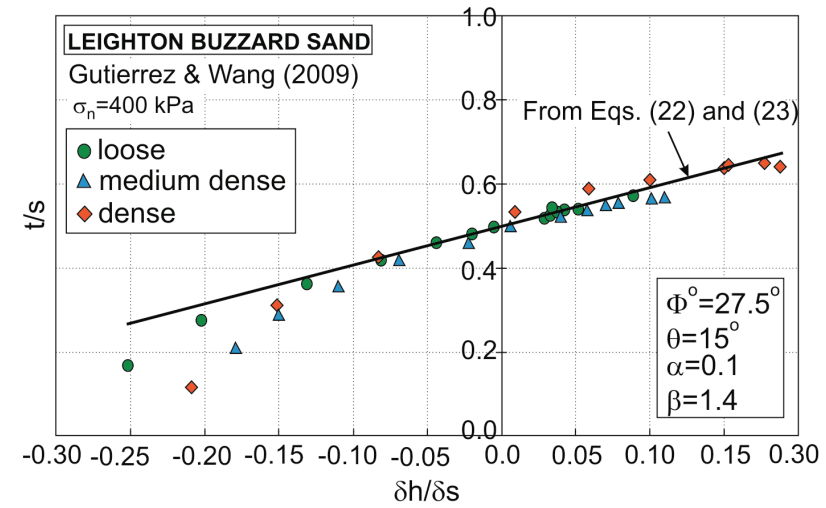

Fig. 6. Stress ratio-dilatancy relationship for Leighton Buzzard sand

Thus, the non-coaxial version of Rowe's stressdilatancy relation [9] can be treated as a special case of equation (23) obtained from the frictional state theory for simple shear.

\section{QUASI-SIMPLE SHEAR}

A new direct shear box was developed at Hokkaido University [28]. Based on extensive experi- mental data, the optimum was assumed to be achieved by lubricating the walls, using a non-rotating loading platen, fixing the opening size to $10-20$ times $D_{50}$ (thickness of the shear band), calculating $\sigma_{n}^{\prime}$ from the measurement force acting below the lower box and using special sample deformation measurement and analysis.

Toyoura and Leighton Buzzard sands were investigated in this newly developed direct shear apparatus. It is assumed that during shearing, quasi-simple shear occurs in the shear band. The relationship between the mobilized angle of the shearing resistance on the horizontal plane $\Phi_{d s}$ and the angle of dilatancy $v$ can be approximated by a straight line [35].

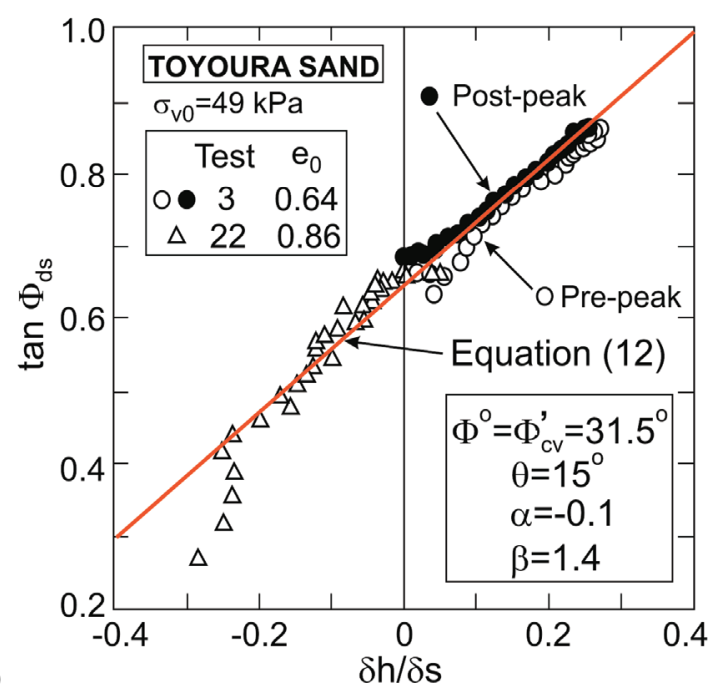

(a)

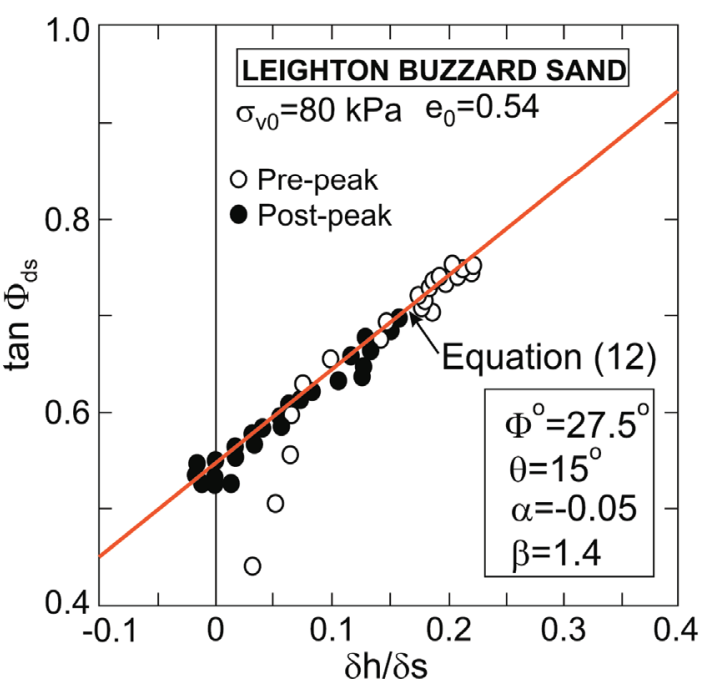

Fig. 7. Relationship between the stress ratio and dilatancy:

(a) Toyoura sand; (b) Leighton Buzzard sand

For the notations used in this paper, $\tan \Phi_{d s}=\tau / \sigma_{n}$ and $\tan v=\delta h / \delta s$. Very good approximations of the experimental data were obtained for the pre-peak and post-peak stages using equation (12) with $\Phi^{o}=31.5^{\circ}$, 
$\theta=15^{\circ}, \alpha=-0.10$, and $\beta=1.4$ for Toyoura sand (Fig. 7a) and with $\Phi^{\circ}=27.5^{\circ}, \theta=15^{\circ}, \alpha=-0.05$, and $\beta=1.4$ for Leighton Buzzard sand (Fig. 7b).

The negative values of $\alpha$ indicate that the residual value of the friction angle is slightly higher than the critical frictional state friction angle $\left(\Phi_{r}^{\prime}>\Phi^{o}\right)$ at quasi-simple shear. The value of $\beta=1.4$ is equal to the value obtained under plane strain conditions.

\section{DIRECT SHEAR}

Four different specimen sizes of a fine poorly graded Toyoura sand were investigated in a direct shear apparatus by $\mathrm{Wu}$ et al. [39]. The specimen sizes (length $\times$ width $\times$ height) were $40 \times 40 \times 20 \mathrm{~mm}$ for small, $120 \times 120 \times 120 \mathrm{~mm}$ for semi-medium, $300 \times 300$ $\times 300 \mathrm{~mm}$ for medium and $800 \times 500 \times 600 \mathrm{~mm}$ for large. For the small and semi-medium samples, constant values of the stress ratio were achieved without changing the sample height. A constant increase in the sample height and changes in the stress ratio were observed for medium and large samples. Analysing the experimental data of the stress ratio and the

(a)

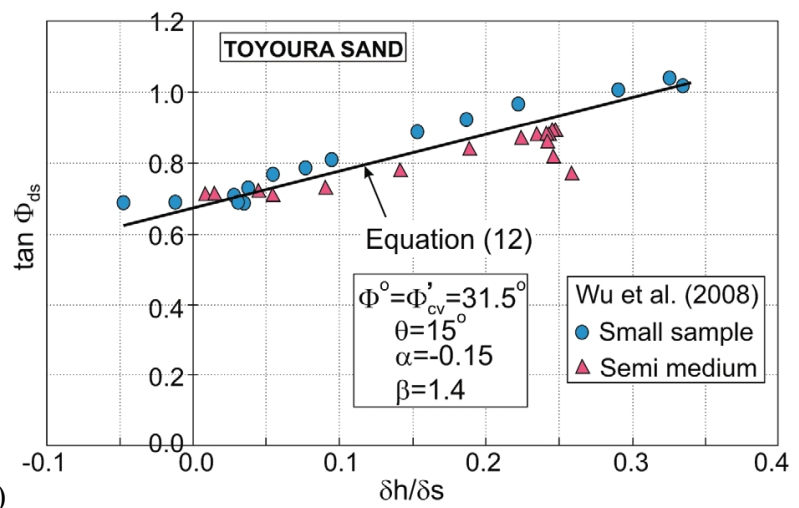

(b)

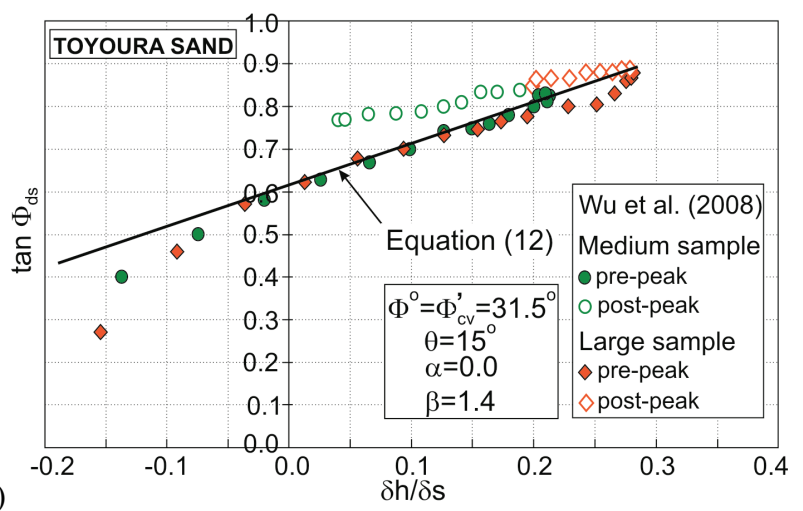

Fig. 8. Influence of the sample size on the $\tan \Phi_{d s}-\delta h / \delta s$ relationship: (a) small and semi-medium samples;

(b) medium and large samples changes in the sample height as functions of the shear displacement [39], the relationships between the stress ratio $\left(\tau / \sigma_{n}^{\prime}\right)$ and $(\delta h / \delta s)$ are shown in Fig. 8.

The best fit of the pre-peak experimental data was obtained using equation (12) for $\Phi^{o}=\Phi_{c v}^{\prime}=31.5^{\circ}$, and $\theta=15^{\circ}$ and with $\alpha=-0.15$ and $\beta=1.4$ for the small and semi-medium samples (Fig. 8a) and $\alpha=0$ and $\beta=1.4$ for the medium and large samples (Fig. 8b).

The Leighton Buzzard sand was tested at a low stress level in a standard Casagrande $60 \mathrm{~mm} \times 60 \mathrm{~mm}$ direct shear box by Scarpelli and Wood [26]. Following the analysis of the experimental dependences of the stress ratio $\left(\tan \Phi_{d s}\right)$ and changes in the sample height on the shear displacement, the relationship between the stress ratio $\left(\tau / \sigma_{n}^{\prime}\right)$ and $(\delta h / \delta s)$ is shown in Fig. 9.

The theoretical relationships were obtained using equation (12) with $\Phi^{o}=27.5^{\circ}, \theta=15^{\circ}, \alpha=-0.3$, and $\beta=2.1$.

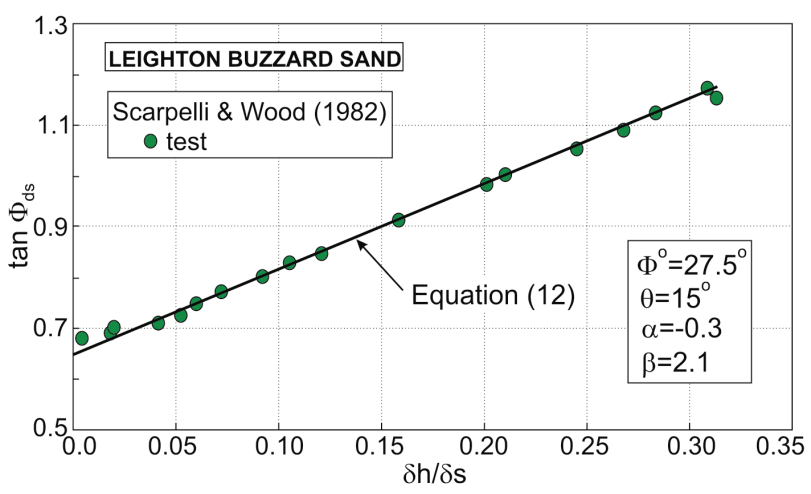

Fig. 9. Relationship between $\tan \Phi_{d s}$ and $(\delta h / \delta s)$ for Leighton Buzzard sand tested by Scarpelli and Wood

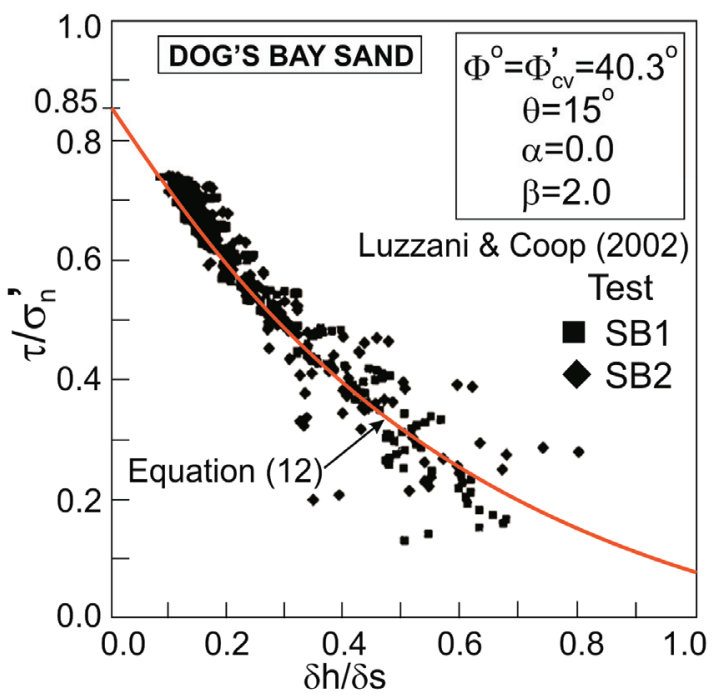

Fig. 10. Stress-dilatancy relationship for shear box tests on Dog's Bay sand 
Luzzani and Coop [17] used a $100 \mathrm{~mm}$ diameter circular box to investigate the relationship between the stress ratio and dilatancy at a high stress level (approximately $800 \mathrm{kPa}$ ) for Dog's Bay sand. Dog's Bay sand is a biogenic carbonate sand with angular, easily broken particles [17], and the critical state friction angle of Dog's Bay sand is $\Phi_{c s}=40.3^{\circ}$ ([16], [29]). The changes in the sample height (volumetric strains) are the result of unknown counterbalancing components of breakage and particle rearrangement. The experimental stress-dilatancy data [17] and theoretical stress-dilatancy relationship obtained from equation (12) with $\Phi^{\circ}=\Phi_{c v}^{\prime}=40.3^{\circ}, \theta=15^{\circ}, \alpha=0$, and $\beta=2.0$ are shown in Fig. 10 .

The line representing the theoretical stress-dilatancy relationship with $\alpha=0$ and $\beta=2.0$ fits the experimental data from shear box tests on Dog's Bay sand well.

\section{RING SHEAR}

Luzzani and Coop [17] also tested Dog's Bay sand in a ring shear apparatus at high stress. Corrections were made for the vertical stress caused by the friction between the soil and the rings and the vertical strain caused by the soil lost through the gap. During shearing, the soil reached an almost constant value of the stress ratio relatively quickly; even the largest strains still showed a slow increase in the stress ratio. In tests, no critical state was reached up to very large shear strains. The experimental stress-dilatancy data and theoretical relationship calculated from equation (12) with $\Phi^{\circ}=\Phi_{c v}^{\prime}=40.3^{\circ}, \theta=15^{\circ}, \alpha=0$, and $\beta=2.0$ are shown in Fig. 11.

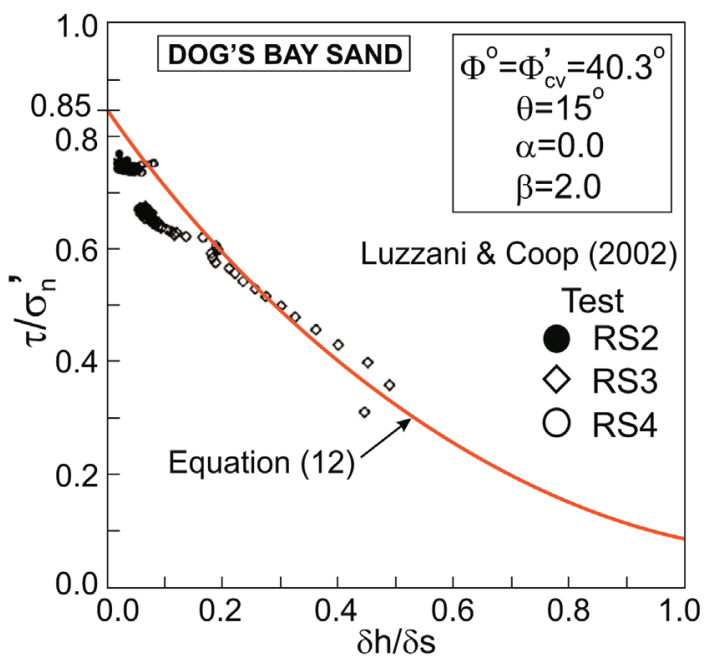

Fig. 11. Stress-dilatancy relationship for ring shear tests on Dog's Bay sand
The theoretical line representing the frictional state theory $(\alpha=0, \beta=2.0)$ fits the experimental data well up to large shear strains. For very large strains, the experimental values of the stress ratio are $10-15 \%$ smaller than those predicted from the frictional state theory. This difference cannot be explained by the experimental data obtained from the ring shear tests.

The unexpectedly high value of $\beta=2.0$ for Dog's Bay sand tested in a shear box and ring shear apparatus at a high stress level is due to the energy consumed and the change in height from the breakage of this sand.

\section{CONCLUSIONS}

The assumptions made in this paper to define the complete stresses and strains in a sample sheared in simple, direct and ring shear apparatuses can be used to calculate the stress-dilatancy relationship for noncohesive soils under drained conditions.

The simple, direct and ring shears can be treated as special cases of plane strain condition.

Assuming that $\Phi^{o}=\Phi_{c v}^{\prime}=\Phi_{r}^{\prime}$, the stress-dilatancy relationships calculated from the frictional state theory and the relationships given by Taylor's and Bolton's equations are similar for a wide range of dilatancy values.

Based on the experimental data analysed in this paper for many non-cohesive soils, the stress-dilatancy relationship calculated from the frictional state theory with $\Phi^{o}=\Phi_{c v}^{\prime}, \theta=15^{\circ}, \alpha=0$, and $\beta=1.4$ is acceptable. Parameters $\alpha$ and $\beta$ indicate not only the soil behaviour but also the sample preparation method, sample size, experimental conditions and faults in the interpretation of the experimental data.

To fully validate the frictional state theory for simple shear conditions of cohesive and non-cohesive soils, further experiments must be conducted in a hollow cylindrical torsional shear apparatus under drained and undrained conditions.

\section{REFERENCES}

[1] AtKinson J.H., LAN W.H.W., Powell J.J.M., Measurement of soil strength in simple shear tests, Can. Geotech. J., 1991, 28, 255-262.

[2] Bardet J.P., Proubet J., A Numerical Investigation of the Structure of Persistent Shear Bands in Granular Media, Geotechnique, 1991, 41, No. 4, 599-613.

[3] BAReither C.A., Benson C.H., EDIL T.B., Reproducibility of direct shear tests conducted on granular backfill materials, Geot. Test. J., 2007, Vol. 31, No. 1, 84-94. 
[4] Bolton M.D., The strength and dilatancy of sands, Geotechnique, 1986, 36, No. 1, 65-78.

[5] BudHu M., Simple shear deformation of sands, Ph.D. thesis, University of Cambridge, 1975.

[6] Cerato A.B., Lutenegger A.L., Specimen size and scale effects of direct shear box tests of sands, Geotechnical Testing Journal, 2006, Vol. 29, No. 6, 1-10.

[7] COLE E.R., The behaviour of soils in the simple shear apparatus. Ph.D. thesis, University of Cambridge, 1967.

[8] Cui L., O'Sullivan C., Exploiting the macro-and-microscale response of an idealised granular material in the direct shear apparatus, Geotechnique, 2006, 56, No. 7, 455-468.

[9] Gutierrez M., Wang J., Non-coaxial version of Rowe's stress-dilatancy relation, Granular Matter., 11, 129-137.

[10] Hosono Y., Yoshimine M., Liquafaction of sand in simple shear condition. Cyclic Behaviour of Soils and Liquafaction Phenomena, Triantafyllidis T. (ed.), Balkema, Rotterdam 2004.

[11] Hosono Y., Yoshimine M., Liquafaction of sand in simple shear condition, Proc. of Int. Conf. on Cyclic Behaviour of Soils and Liquafaction Phenomena, Bohun, Germany, March 31th-April 2nd 2004.

[12] Hong NAm N., KoseKI J., Modelling quasi-elastic deformation properties of sand, Deformation Characteristics of Geomaterials, IS - Lyon 2003, 275-283.

[13] Jewell R.A., Wroth C.P., Direct shear tests on reinforced sand, Geotechnique, 1987, 37, No. 1, 53-68.

[14] Likos W.J., Wayllace A., Godt J., Lu N., Modified Direct Shear Apparatus for Unsaturated Sands at Low Suction and Stress, Geotechnical Testing Journal, 2010, 33, No. 4, 286-298.

[15] Lings M.L., DiETZ M.S., An improved direct shear apparatus for sand, Geotechnique, 2004, 54, No. 4, 245-256.

[16] Lopez-Querol S., CoOP M.R., Drained cyclic behaviour of loose Dog's Bay sand, Geotechnique, 2012, 62, No. 4, 281-289.

[17] LuZZANI L., CoOP M.R., On the relationship between particle breakage and the critical state of sands, Soils and Foundations, 2002, Vol. 42, No. 2, 71-82.

[18] Milatz M., Grabe J., A new simple shear apparatus and testing method for unsaturated sands. Geotech. Testing J., 2015, Vol. 38, No. 1, 9-22.

[19] Meehan C.L., Brandon T.L., Duncan J.M., Measuring Drained Residual Strengths in the Bromhead Ring Shear, Geotechnical Testing Journal, 2007, 30, No. 6, 466-473.

[20] Ochiai H., Stress condition within simple shear test specimen, Reports of the Faculty of Engineering, Nagasaki University, No. 12, February, 1979, 57-63.

[21] ODA M., On stress-dilatancy relation of sand in simple shear test, Soils and Foundations, 1975, Vol. 15, No. 2, 17-29.

[22] Palmeira E.M., Milligan G.W.E., Scale Effects in Direct Shear Tests on Sand, Proc. of the $12^{\text {th }}$ ICSMGE, 1989, Vol. 1, No. 1, 739-742.

[23] Potts D.M., Dounias G.T., Vaughan P.R., Finite element analysis of the direct shear box test, Geotechnique, 1987, Vol. 37, No. 1, 11-23.
[24] Qiu Y.-Y., Tatsuoka F., Uchimura T., Constant Pressure and constant volume direct shear tests on reinforced sand, Soil and Foundations, 2000, 40, No. 4, 1-17.

[25] Sassa K., Wang G., Fukuaka H., Performing Undrained Shear Tests on Saturated Sands in a New Intelligent Type of Ring Shear Apparatus, Geotechnical Testing Journal, 2003, 26, No. 3, 257-265.

[26] SCARPElli G., WoOD D.M., Experimental observations of shear band patterns in direct shear tests, IUTAM Conference on Deformation and Failure of Granular Materials. Delft/31-Aug.-3 Sept. 1982, 473-484.

[27] Shibuya S., Koseki J., Kawaguchi T., Recent developments in deformation and strength testing of geomaterials, Deformation Characteristics of Geomaterials. Di Benedetto et al. (eds.), Taylor \& Francis Group, London 2005, 3-28.

[28] Shibuya S., Mitachi T., Tamate S., Interpretation of direct shear box testing of sands as quasi-simple shear, Geotechnique, 1997, 47, No. 4, 769-790.

[29] ShIPTON B., Coop M.R., On the compression behaviour of reconstituted soils, Soils and Foundations, 2012, 52, No. 4, $668-681$.

[30] Smoltczyk U. ed., Geotechnical Engineering Handbook. Vol. 1: Fundamentals, Ernest \& Sohn. A Wiley Company, Berlin 2002.

[31] Szypcio Z., Stress-dilatancy for soils. Part I: The frictional state theory, Studia Geotechnica et Mechanica, 2016, Vol. 38, No. 4, 51-57.

[32] Szypcio Z., Stress-dilatancy for soils. Part II: Experimental validation for triaxial tests, Studia Geotechnica et Mechanica, 2016, Vol. 38, No. 4, 59-65.

[33] Szypcio Z., Stress-dilatancy for soils. Part III: Experimental validation for the biaxial conditions, Studia Geotechnica et Mechanica, 2017, Vol. 39, No. 1, 73-80.

[34] Tang Y.X., Hanzawa H., Yasuhara K., Direct shear and direct simple shear tests results on Japanese marine clay, Pre-failure Deformation of Geomaterials. Balkema, Rotterdam 1995, 107-112.

[35] TAYLOR D.W., Fundamentals of Soil Mechanics, John Wiley \& Sons, New York 1948.

[36] TAYLOR D.W., A direct test with drainage control, Symp. on Direct Shear Testing of Soils, ASTM Special Techn. Publ., 1952, No. 131, 63-74.

[37] Wijewickreme D., Dabeet A., Byrne P., Some Observations on the State of Stress in the Direct Simple Shear Test Using Discrete Element Analysis, Geotechnical Testing Journal, 2013, 36, No. 2, 292-298.

[38] Wood D.M., Soil behaviour and critical state soil mechanics, Cambridge University Press, 1990.

[39] Wu P.-K., Matsushima K., Tatsuoka F., Effects of Specimen Size and Some Other Factors on the Strength and Deformation of Granular Soil in Direct Shear Tests, Geotechnical Testing Journal, 2008, 31, No. 1, 45-64.

[40] Yan W.M., Particle Elongation and Deposition Effect to Macroscopic and Microscopic Responses of Numerical Direct Shear Tests, Geotechnical Testing Journal, 2010, 34, No. 3, 238-249. 